Vol. 6, n² | 2002

Varia

\title{
Juger les juges. Du Moyen Âge au Conseil Supérieur de la Magistrature
}

Association française pour l'histoire de la justice, Collection histoire de la justice $N^{\circ} 12$, Paris, La Découverte, 2000, 289 p., ISBN 2-11-004431-2

Françoise Briegel

\section{(2) OpenEdition}

Journals

Édition électronique

URL : https://journals.openedition.org/chs/444

DOI : $10.4000 /$ chs.444

ISSN : 1663-4837

Éditeur

Librairie Droz

Édition imprimée

Date de publication : 1 décembre 2002

Pagination : 141-144

ISBN : 2-600-00812-8

ISSN : 1422-0857

Référence électronique

Françoise Briegel, « Juger les juges. Du Moyen Âge au Conseil Supérieur de la Magistrature », Crime, Histoire \& Sociétés / Crime, History \& Societies [En ligne], Vol. 6, n² | 2002, mis en ligne le 19 février 2009, consulté le 23 mars 2022. URL : http://journals.openedition.org/chs/444 ; DOI : https://doi.org/ $10.4000 /$ chs. 444

Ce document a été généré automatiquement le 23 mars 2022.

(C) Droz 


\section{Juger les juges. Du Moyen Âge au Conseil Supérieur de la Magistrature}

Association française pour l'histoire de la justice, Collection histoire de la justice $\mathrm{N}^{\circ} 12$, Paris, La Découverte, 2000, 289 p., ISBN 2-11-004431-2

\section{Françoise Briegel}

\section{RÉFÉRENCE}

Juger les juges. Du Moyen Âge au Conseil Supérieur de la Magistrature, Association française pour l'histoire de la justice, Collection histoire de la justice $\mathrm{N}^{\circ} 12$, Paris, La Découverte, 2000, 289 p., ISBN 2-11-004431-2.

1 Fruit d'un colloque organisé en 1998, par l'Association française pour l'histoire de la justice, ce volume réunit les contributions d'une quinzaine de spécialistes sur le thème de la responsabilité du juge depuis le Moyen Âge jusqu'à la période contemporaine. Ces articles de grande qualité et richement documentés ne feront malheureusement pas tous l'objet d'un compte rendu étoffé.

Dans ce vaste tableau chronologique un questionnement commun sous-tend toutes les contributions: quelle est l'autonomie des juges vis-à-vis du pouvoir politique? Les réflexions proposées dans cet ouvrage montrent le difficile équilibre, voire la tension qui existe entre la discipline interne dispensée par les juges au sein de l'institution judiciaire et celle que le pouvoir politique cherche à leur imposer. Cet ouvrage propose une réflexion qui fait écho à l'actualité la plus récente car la fraîche indépendance judiciaire conduit les juges à déférer certains hommes politiques devant les tribunaux, ce qui constitue une nouveauté au regard des siècles passés. Ce colloque pourra sans nul doute apporter une réponse à un récent article de Jacques Gaillard, paru dans le Monde du 6 décembre 2001, qui s'interrogeait sur la nécessité de juger les juges. Ces articles soulignent comment cette indépendance judiciaire vis-à-vis de l'autorité politique s'est lentement construite. 
3 À partir de textes et de l'iconographie, Robert Jacob montre qu'au Moyen Âge le fondement de la responsabilité des juges est ancré dans les domaines symboliques et religieux. Suite au serment d'entrée en fonction, réitéré annuellement, la faute des juges relève du parjure. Alors que les différends entre les justiciables et leurs juges étaient résolus par un combat judiciaire, la procédure inquisitoire introduit un appel devant les juridictions supérieures pour régler ces conflits. La sanction suprême est rendue par le Parlement de Paris. Cette responsabilité médiévale, mise en scène par l'iconographie des salles de justice qui représente les erreurs judiciaires, insiste alors sur la dimension faillible du juge. Cette ostentation médiévale, par la suite occultée, ne discrédite pas la justice puisque cette transparence assure une fonction de légitimation. Claude Gauvard, quant à elle, nous plonge dans la pratique du Parlement de Paris. Sur la base des procès criminels, elle s'interroge sur le statut du juge à la fin du Moyen Âge. Dans les mises en cause des juges, peu de sentences de destitutions sont rendues et lorsqu'elles les sanctionnent, elles n'entravent pas la carrière des magistrats. Les amendes honorables qui visent à rétablir l'honneur de la partie lésée, sanctionnent la personne privée du juge, sans affaiblir la fonction judiciaire. L'office du juge, construit davantage sur la pratique que sur la théorie, s'est nourri des absences de jugements de juges, puisque les sanctions du Parlement sont peu nombreuses. Les lettres de rémission (justice retenue) servent à protéger le juge et à préserver la partie adverse. Dès 1454, le statut est assez solide pour que l'appel, auparavant limité aux causes civiles soit possible pour les sentences de mort. Ainsi le statut du juge s'est construit sur un équilibre entre le refus par le Parlement d'approuver des conduites iniques de ses membres et le refus de discréditer la justice. L'amende honorable et les lettres de rémission ont donc été des instruments dans le processus de renforcement de la justice royale.

4 Sylvain Soleil constate que sous l'Ancien Régime, aucune instance spécifique et extérieure à la magistrature n'intervient comme organe de contrôle disciplinaire. Les cours souveraines exercent une discipline à caractère hiérarchique, qui règlent les infractions relevant d'obligations morales et légales. Cette discipline interne vise à soustraire le corps d'une intervention extérieure puisque selon une procédure d'enquête, l'action est entamée devant le Procureur général du Parlement. Comme au Moyen Âge, l'amende honorable sanctionne les juges considérés comme responsables. Aux $\mathrm{XV}^{\mathrm{e}}$ et $\mathrm{XVI}{ }^{\mathrm{e}}$ siècles, l'inamovibilité, l'hérédité et la vénalité des charges dépossèdent le roi du contrôle judiciaire et automonisent le corps. Au XVII siècle, une structure corporative règle les cas d'indiscipline et le roi ne sanctionne que les cas les plus graves. L'équilibre entre un contrôle disciplinaire interne à la magistrature et le pouvoir royal se crée. Dans le prolongement de l'étude précédente, Arlette Lebigre, relève que la discipline corporative, où les juges sont jugés par leurs pairs est remise en cause dès 1560. Aux États Généraux de 1614, les cahiers de doléances s'élèvent contre la corruption des magistrats et réclament l'intervention du roi afin de sanctionner les abus. Au début du XVII ${ }^{\mathrm{e}}$ siècle, les tentatives royales de légiférer les magistrats ne se traduisent pas par véritable droit royal de sanction. Il faut attendre la deuxième moitié du XVII ${ }^{e}$ siècle, pour que Colbert installe un véritable contrôle. Aux Grands Jours d'Auvergne en 1665, quelques retentissantes condamnations marquent le début d'un droit de regard sur le corps judiciaire. L'autorité ministérielle de contrôle des magistrats se régularise à la fin $\mathrm{du} \mathrm{XVII}^{\mathrm{e}}$ siècle et cette tendance s'accentue pour le XVIII ${ }^{e}$ siècle. Concernant la responsabilité des juges prévue par l'Ordonnance civile de 1667, Catherine Methy s'arrête sur deux moyens légaux qui sanctionnent les juges: la 
récusation et la prise à partie. Légiférées par les titres 24 et 25 de l'Ordonnance ces actions peuvent être engagées complémentairement, mais Catherine Methy montre que les parties lésées se tournent vers la récusation qui est moins complexe. Celle-ci met en cause l'individu alors que la prise à partie est l'aveu d'une sanction civile pour faute et peut annuler la procédure dans sa totalité. L'Ordonnance de 1667 traduit ainsi une transition entre deux conceptions de la responsabilité, l'une individuelle et l'autre qui touche la fonction judiciaire. Jean-Louis Halpérin introduit la notion de responsabilité du juge au regard des troubles parlementaires du XVIII siècle. Confrontée au problème de l'inamovibilité des charges, la justice retenue a perdu de son pouvoir répressif face à ses officiers. En réponse au problème de l'insoumission des parlements concernant le refus des sacrements, Louis $\mathrm{XV}$, choisit la suppression des chambres et donc le rachat des offices. Maupéou instaure une nouvelle législation en 1770, afin de statuer sur des cas de privation d'office qui sont alors du ressort du Conseil du roi. Le parlement de Paris refuse l'enregistrement de ce nouveau règlement. La discussion s'engage sur le terrain de la légalité de la réforme. Dans l'espace public, s'opposent alors les thèses pro et anti parlementaires. La bataille tourne en faveur des pro-parlementaristes puisque Maupéou abandonne l'idée de forfaiture en décidant de racheter les offices supprimés. Cette crise parlementaire montre que la tentative d'instaurer un délit de forfaiture jugé par le roi seul n'était plus concevable dans un climat de légalité fondée sur la séparation des pouvoirs. Elle illustrait également l'hostilité des juges à la révocation de leur charge par le pouvoir exécutif.

Dans le prolongement des études précédentes qui illustraient le difficile équilibre entre l'indépendance et la soumission des juges au pouvoir politique tant au niveau disciplinaire que politique, Jean-Jacques Clère s'intéresse à la renaissance, au XIX ${ }^{\mathrm{e}}$ siècle, d'une auto-discipline qui repose sur la notion de corps et dont les règles garantissent la soumission des juges aux devoirs de la profession. À la Révolution, la création du ministère de la Justice, en 1791, place dans les mains du ministre certains pouvoirs disciplinaires qui rentrent en conflit avec l'idéal de séparation des pouvoirs révolutionnaires. Il y a une interférence du pouvoir politique dans la sphère judiciaire. Le nouveau régime de l'An VIII, voit la disparition de l'élection des juges et leur nomination par le gouvernement. Le pouvoir disciplinaire est transféré dans les mains $\mathrm{du}$ politique. Sous Napoléon, le judiciaire se voit réattribué ses compétences disciplinaires. Précisé sous l'Empire, le texte légal sert de référence pour tout le XIX ${ }^{\mathrm{e}}$ siècle et cinq niveaux de juridictions disciplinaires sont prévus. La loi de 1883, simplifie cet emboîtement juridictionnel en supprimant les niveaux intermédiaires et instaure la Cour de Cassation qui représente le premier Conseil Supérieur de la Magistrature. François Fourn étudie le cas d'Étienne Cabet, qui comparaît en 1834 devant la cour d'assise et montre que ce procès pour liberté d'opinion politique est emblématique de la soumission du judiciaire au pouvoir politique. En étudiant les jugements des magistrats opposés au coup d'État de 1851 devant une commission mixte (juridiction d'exception pénale créée par simple circulaire), Renée Martinage souligne l'épuration du corps judiciaire. Cette commission mixte prononce quelques 26000 sentences dont un tiers de déportations en Algérie. Face à cette répression les magistrats se rallient au bonapartisme et renient leurs convictions républicaines. Lors de la proclamation de la $\mathrm{III}^{\mathrm{e}}$ République, les magistrats siégeant dans les commissions mixtes sont destitués. Une autre forme d'épuration s'instaure, elle passe par des décrets de destitution, de révocations, etc. Renée Martinage constate que lors des changements politiques importants, il s'opère une épuration politique dans l'appareil judiciaire qui peut 
prendre des formes sommaires, sans jugement. Anne-Marie Mallet, montre que le débat parlementaire autour de la Loi de 1883, modifie l'organisation judiciaire et précise le statut du personnel. Cette loi n'envisage la responsabilité des juges que selon un mode disciplinaire. Émerge alors l'idée d'un conseil de discipline de la magistrature.

La question du jugement disciplinaire des juges est au cœur de l'indépendance de la justice, selon Alain Bancaud, et Jean-Paul Jean. Au début du XXe siècle, les tentatives de créer une inspection des services judiciaires sont nombreuses. En 1962, la création d'un inspecteur général qui aura une fonction permanente s'ouvre dès 1965 à d'autres inspecteurs. Cette inspection s'inscrit dans l'affirmation du pouvoir central sur la justice. Elle joue explicitement le jeu du ministère pour s'imposer à l'intérieur de la Chancellerie. La création d'un corps d'inspecteurs s'inscrit dans un mouvement d'affaiblissement de la cour de cassation au profit du personnel de la Chancellerie et assure la continuité judiciaire face à l'instabilité politique. Pour clore ces contributions sur la responsabilité des juges, Dominique Commaret, s'arrête sur le Conseil Supérieur de la Magistrature qui institue un principe de non-ingérence politique dans les décisions judiciaires. Ce Conseil statue sur l'impartialité des juges et pose des règles morales. En cas d'irrégularité la sanction condamne l'État et non la personne du juge. Les relations économiques ou contractuelles, les activités associatives, l'engagement religieux ou politique des juges sont sous la loupe du Conseil. La justice est considérée comme une dette vis-à-vis de la société où le mal-jugé n'est pas sanctionné.

Deux articles clôturent cet ouvrage, celui de Ghislain Waterlot concernant deux écrits de Voltaire sur la justice (Le prix de la justice et de l'humanité et le Commentaire sur l'Esprit des lois, 1777) et celui de Jean-François Chassaing qui traite de l'histoire de la présomption d'innocence.

\section{AUTEUR}

FRANÇOISE BRIEGEL

Université de Genève, fbriegel@libertysurf.fr 